\title{
Development of radiotherapy for liver cancer
}

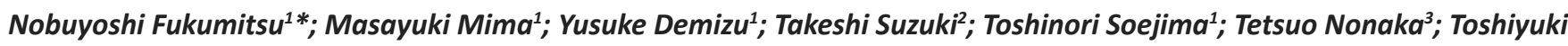 \\ Okumura $^{3}$; Hideyuki Sakurai ${ }^{3}$ \\ ${ }^{1}$ Department of Radiation Oncology, Kobe Proton Center, Japan \\ ${ }^{2}$ Department of Anesthesiology, Kobe Proton Center, Japan \\ ${ }^{3}$ Department of Radiation Oncology, University of Tsukuba, Japan
}

\begin{abstract}
*Corresponding Author(s): Nobuyoshi Fukumitsu, Department of Radiation Oncology, Kobe Proton Center, 1-6-8, Minatoshima Minamimachi, Chuoku, Kobe, Japan
\end{abstract}

Tel: 81-78-335-8001, Fax: 881-78-335-8005,

Email: fukumitsun@yahoo.co.jp

Received: Mar 06, 2018

Accepted: May 10, 2018

Published Online: May 16, 2018

Journal: Annals of Gastroenterology and the Digestive System Publisher: MedDocs Publishers LLC

Online edition: http://meddocsonline.org/

Copyright: (C) Fukumitsu N (2018). This Article is distributed under the terms of Creative Commons Attribution 4.0 International License

Keywords: Liver cancer; Radiotherapy; Three-dimensional conformal radiotherapy; Stereotactic body radiotherapy; Particle beam therapy

\section{Introduction}

Accurate localized diagnosis of liver cancers was difficult until the 1970s, and significance of radiotherapy for liver cancers is only palliative because it was difficult to suppress the damage to the radiosensitive liver. However, as Computed Tomography (CT) became widespread for treatment planning in the1980s, the localized diagnosis of lesions became easy, therefore, treatment could be performed while reducing liver damage. In recent years, radiotherapy is recognized to be one of the curative treatment technique for liver cancers. Here we outline contemporary liver cancer radiotherapy.

\section{Abstract}

Radiotherapy for liver cancers used to be palliative treatment. Currently, three-dimensional conformal radiotherapy, stereotactic body radiotherapy, and particle beam therapy using proton beams and carbon ion beams are used for the treatment of liver cancers. Radiotherapy for liver cancers has become to curative treatment due to the various technical progress. Radiotherapy can accomplish safe and effective treatment for liver cancers.

\section{Three-dimensional conformal radiotherapy (3D-CRT)}

X-rays are usually used in 3D-CRT. Radiation is beamed from a direction perpendicular to the body axis in most cases; however it can be delivered from other directions by turning the patients' bed. One advantage of 3D-CRT is that it means the shape of the irradiation field can be set 3-dimensionallyenablingdelivery of enough radiation to the target, accomplishing conformal beam irradiation to the target, while suppressing the irradiation dose to important tissue around the tumor. 3D-CRT is used in cases when other treatments are not available due to Portal Vein Tumor Thrombosis (PVTT), unrespectability, or some complications.

Cite this article: Fukumitsu N, Mima M, Demizu Y, Suzuki T, Soejima T, et al. Development of radiotherapy for liver cancer. Ann Gastroenterol Dig Syst. 2018; 1: 1005. 
A total irradiation dose around 45-50 Gyat a daily dose of 2 Gyisoften reported. There have been many prospective studies in which the response rate was $30-80 \%$ and the 1-year survival rate was $25-50 \%$ for PVTT or for Inferior Vena Cava Thrombosis (IVCT) patients [1-4]. Prospective and retrospective studies of unrespectable cases have shown that the survival of patients who received Transarterial Chemoembolization (TACE) with radiotherapy was longer than patients who received only TACE, and a meta-analysis study also proved higher response and survival rates in the TACE-with-radiotherapy group ${ }^{5}$.As for adverse effects, it has been reported that radiotherapy can be performed safely, with only elevation of the total bilirubin value in many cases [5].

\section{Stereotactic body radiotherapy (SBRT)}

SBRT has a feature for treating small tumors. To condense radiation and to hit the target accurately, it meets 3 criteria: (1) higher dose to be delivered to a small target in a short term from multiple directions using a linear accelerator, (2) geometrical accuracy finer than $5 \mathrm{~mm}$ in every treatment session, (3) immobilizing the patient in position and countermeasures for respiratory movement. Various device to fix the body and highly accurate position matching system are required.

A total dose of 30-50 Gy at a daily dose of 6-15 Gy to the tumor less than $5 \mathrm{~cm}$ is usually performed. Even when the lesions treated are difficult to cure by other local treatment, relatively good outcomes are reported, such as a response rate of $50-85 \%$, 1 -year local control rate of $65-100 \%, 1$-year overall survival rate $50-90 \%$, 2-year local control rate of 90-95\% [6-10]. Serious liver damage from the technique is very rare.

\section{Particle beam therapy}

Particle beam therapy uses high-energy protons carbon ions. In contrast to X-rays, accelerated charged protons and carbon ions release their maximum energy just before they stop, creating a steep peak of energy called the Bragg-peak. Furthermore, regulating the dose distribution in the depth direction can be accomplished by mixing different energy beams together, called the spread-out Bragg peak. Figure 1 shows the concepts of $X$ rays and particle beams and Figure 2 shows dose distribution of proton beam therapy for liver cancers. Although the indication for treatment is similar to X-ray radiotherapy, particle beam therapy can treat more advanced disease condition.

There are many reports of a total dose of radioactivity 6070 Gray Equivalent (GyE) at a daily dose of 2-6 GyE for proton beam therapy. Dose escalation studies of carbon ion beam therapy from 49.5 GyE to 79.5 GyE reveal that a total dose of 72 GyE at a daily dose of 4.8 GyEis ideal [11], and short term treatment protocols, such as approximately $50 \mathrm{GyE}$ in 1-2 weeks, is also done. Many previous studies treated patients in Child-Pugh classification A or B whom it was difficult to give other local treatment $[12-15,16]$. In addition, PVTT, IVCT or a huge tumor can be applied $[17,18]$. Excellent local control with extremely rare adverse effects can be achieved.

\section{Conclusion}

Radiotherapy for liver cancer has been developed due to technical progress such as the improvement of imaging, threedimensional treatment planning, reproducible irradiation technique, and precise management of the irradiation treatment system. In the daily clinic, more number of patients has come to hospital to ask for radiotherapy.

On the other hand, clinical history of radiotherapy for liver cancer is at most 20-30 years. Thus, there is still no firm consensus about indications for this treatment. Although there are many reports about effectiveness and safety, they do not refer to evidence-rich data for deciding the criteria for radiotherapy. More evidence-rich data is required in order to popularize radiotherapy for liver cancer to help these patients in future.

Figures

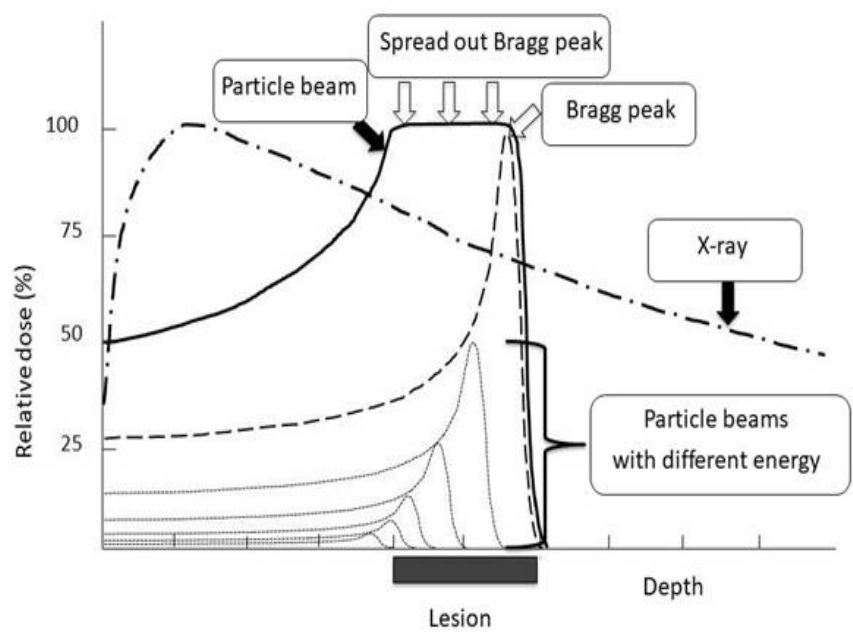

Figure 1: Relative dose and depth (comparison of X-rays and particle beams).

\section{Before treatment}
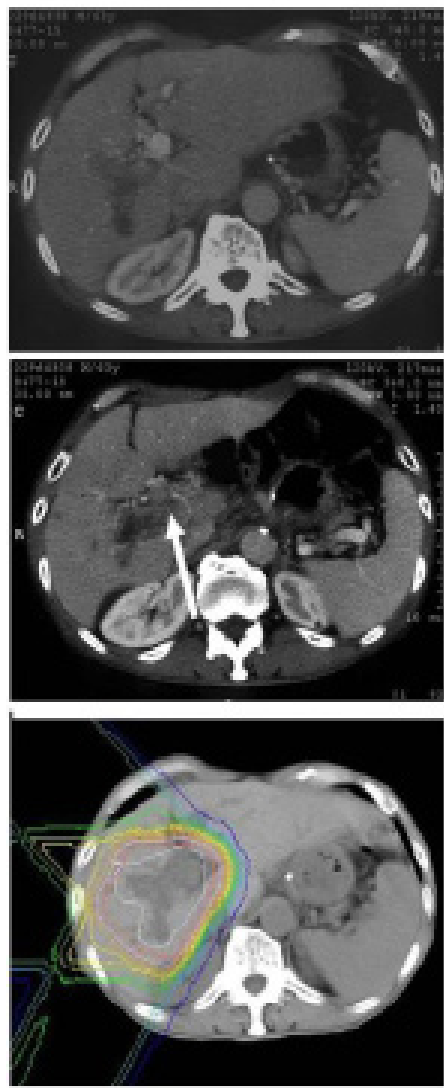

Figure 2: HCC patient. 63 years old man having HCC with PVTT (Vp4). Complete response and re-canalization was seen 18 months after PBT (arrow).

\section{8 months after PBT}

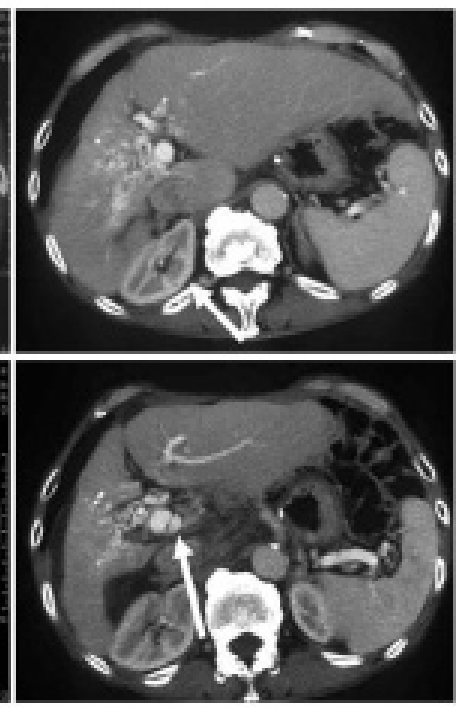




\section{References}

1. Shirai S, Sato M, Suwa K, Kishi K, Shimono C, Sonomura $\mathrm{T}$, et al. Feasibility and efficacy of single photon emission computed tomography-based three-dimensional conformal radiotherapy for hepatocellular carcinoma $8 \mathrm{~cm}$ or more with portal vein tumor thrombus in combination with transcatheter arterial chemoembolization. Int J Radiat Oncol Biol Phys. 2010; 76: 1037-1044.

2. Lin CS, Jen YM, Chiu SY, Hwang JM, Chao HL, Lin HY, et al. Treatment of portal vein tumor thrombosis of hepatoma patients with either stereotactic radiotherapy or three-dimensional conformal radiotherapy. Jpn J Clin Oncol. 2006; 36: 212-217.

3. Ishikura S, Ogino T, Furuse J, Satake M, Baba S, Kawashima $\mathrm{M}$, et al. Radiotherapy after transcatheter arterial chemoembolization for patients with hepatocellular carcinoma and portal vein tumor thrombus. American journal of clinical oncology. 2002; 25: 189-193.

4. Huang CJ, Lian SL, Chen SC, Wu DK, Wei SY, Huang MY, et al. External beam radiation therapy for inoperable hepatocellular carcinoma with portal vein thrombosis. The Kaohsiung journal of medical sciences. 2001; 17: 610-614.

5. Meng MB, Cui YL, Lu Y, She B, Chen Y, Guan YS, et al. Transcatheter arterial chemoembolization in combination with radiotherapy for unresectable hepatocellular carcinoma: a systematic review and meta-analysis. Radiother Oncol. 2009; 92: 184-194.

6. Tse RV, Hawkins M, Lockwood G, Kim JJ, Cummings B, Knox J, et al. Phase I study of individualized stereotactic body radiotherapy for hepatocellular carcinoma and intrahepatic cholangiocarcinoma. J Clin Oncol. 2008; 26: 657-664.

7. Goodman KA, Wiegner EA, Maturen KE, Zhang Z, Mo $Q$, Yang $G$, et al. Dose-escalation study of single-fraction stereotactic body radiotherapy for liver malignancies. Int J Radiat Oncol Biol Phys. 2010; 78: 486-493.

8. Andolino DL, Johnson CS, Maluccio M, Kwo P, Tector AJ, Zook J, et al. Stereotactic body radiotherapy for primary hepatocellular carcinoma. Int J Radiat Oncol Biol Phys. 2011; 81: e447-453.

9. Louis C, Dewas S, Mirabel X, Lacornerie T, Adenis A, Bonodeau $F$, et al. Stereotactic radiotherapy of hepatocellular carcinoma: preliminary results. Technology in cancer research \& treatment. 2010; 9: 479-487.
10. Choi BO, Jang HS, Kang KM, Lee SW, Kang YN, Chai GY, et al. Fractionated stereotactic radiotherapy in patients with primary hepatocellular carcinoma. Jpn J Clin Oncol. 2006; 36: 154-158.

11. Kato H, Tsujii H, Miyamoto T, Mizoe JE, Kamada T, Tsuji $\mathrm{H}$, et al. Results of the first prospective study of carbon ion radiotherapy for hepatocellular carcinoma with liver cirrhosis. Int J Radiat Oncol Biol Phys. 2004; 59: 1468-1476.

12. Kawashima M, Furuse J, Nishio T, Konishi M, Ishii $H$, Kinoshita T, et al. Phase II study of radiotherapy employing proton beam for hepatocellular carcinoma. J Clin Oncol. 2005; 23: 1839-1846.

13. Bush DA, Kayali Z, Grove R, Slater JD. The safety and efficacy of high-dose proton beam radiotherapy for hepatocellular carcinoma: a phase 2 prospective trial. Cancer. 2011; 117: 3053-3059.

14. Imada H, Kato H, Yasuda S, Yamada S, Yanagi T, Kishimoto $\mathrm{R}$, et al. Comparison of efficacy and toxicity of short-course carbon ion radiotherapy for hepatocellular carcinoma depending on their proximity to the porta hepatis. Radiother Oncol. 2010; 96: 231-235.

15. Fukumitsu N, Sugahara S, Nakayama H, Fukuda K, Mizumoto $\mathrm{M}$, Abei $\mathrm{M}$, et al. A prospective study of hypofractionated proton beam therapy for patients with hepatocellular carcinoma. Int J Radiat Oncol Biol Phys. 2009; 74: 831-836.

16. Mizumoto M, Okumura T, Hashimoto T, Fukuda K, Oshiro $\mathrm{Y}$, Fukumitsu N, et al. Proton beam therapy for hepatocellular carcinoma: a comparison of three treatment protocols. Int J Radiat Oncol Biol Phys. 2011; 81: 1039-1045.

17. Komatsu S, Fukumoto T, Demizu Y, Miyawaki D, Terashima K, Sasaki R, et al. Clinical results and risk factors of proton and carbon ion therapy for hepatocellular carcinoma. Cancer. 2011; 117: 4890-4904.

18. Sugahara S, Oshiro Y, Nakayama H, Fukuda K, Mizumoto $M$, Abei $M$, et al. Proton beam therapy for large hepatocellular carcinoma. Int J Radiat Oncol Biol Phys. 2010; 76: 460-466. 